\title{
Use of over-the-scope-clip (OTSC) improves outcomes of high-risk adverse outcome (HR-AO) non-variceal upper gastrointestinal bleeding (NVUGIB)
}

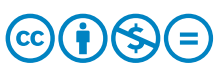

\author{
Authors \\ Jing Hieng $\mathrm{Ngu}^{1,2}$ \\ Institutions \\ 1 Singapore General Hospital, Singapore \\ 2 Duke Graduate School of Medicine - National University \\ of Singapore, Singapore \\ 3 Hospital Costa del Sol, Marbella, Malaga, Spain
}

Ravishankar Asokkumar ${ }^{1}$, Roy Soetikno ${ }^{1,2}$, Andres Sanchez-Yague ${ }^{3}$, Lim Kim Wei ${ }^{1}$, Ennaliza Salazar ${ }^{1}$,

submitted 6.2.2018

accepted after revision 13.4 .2018

\author{
Bibliography \\ DOI https://doi.org/10.1055/a-0614-2390 | \\ Endoscopy International Open 2018; 06: E789-E796 \\ (c) Georg Thieme Verlag KG Stuttgart · New York \\ ISSN 2364-3722
}

Corresponding author

Roy Soetikno, MD, MS, MSM, Advanced GI Endoscopy, 2450

Hospital Drive, Suite 211, Mountain View, CA 94040

soetikno@earthlink.net

\section{ABSTRACT}

Background and study aims Endoscopic treatment of non-variceal upper gastrointestinal bleeding (NVUGIB) with high-risk adverse outcome (HR-AO) features has a high risk of failure. We studied the safety and efficacy of over-the-scope clips (OTSC) to treat these lesions.
Patients and methods We included patients who were treated using OTSC for NVUGIB from January 2015 to October 2017. We studied rebleeding and mortality rates and used the Rockall data and our institution's prior data for comparison. We used descriptive and chi-square statistics. Results We studied 18 patients with 19 bleeding lesions: 9 (47\%) duodenal ulcers, 4 (21\%) Dieulafoy's lesion, 3 (16\%) gastric ulcer, and 3 (16\%) bleeding after gastric biopsy, gastric polypectomy and endoscopic ultrasound-guided fineneedle aspiration of peri-gastric mass. We applied OTSC as the first-line treatment in $10(53 \%)$ and as the second-line treatment in 9 (47\%) bleeding lesions. Continued bleeding after OTSC occurred in six patients, but we treated it successfully and achieved complete hemostasis in all patients. We found OTSC use significantly decreased ( $0 \%$ vs. $53 \%, P<$ 0.01 ) and reduced ( $0 \%$ vs. $24 \%, P=0.08)$ the rebleeding rate in our high-risk $(R S \geq 8)$ and intermediate-risk ( $R S=$ 4 -7) Rockall score patients as compared to the rates reported by the Rockall study, respectively. When compared to our institution's prior study, we found a decrease in the rebleeding rate with OTSC ( $0 \%$ vs. $21 \%, P=0.06$ ) in our intermediate-to-high risk Rockall score patients ( $R S \geq 4)$. There was no difference in mortality rates as compared to both control studies.

Conclusion Use of OTSC is safe, efficacious and appears superior to standard treatment for HR-AO NVUGIB. OTSC should be considered as first-line treatment for HR-AO bleeding.

\section{Introduction}

Despite significant advancements in endoscopic hemostatic devices, treatment of nonvariceal upper gastrointestinal bleeding (NVUGIB) with high-risk adverse outcome (HR-AO) features has remained challenging. HR-AO bleeding is defined as bleeding due to a large-caliber (>2 $\mathrm{mm}$ ) artery; bleeding located within the major arterial territories (left gastric and gastroduodenal artery); bleeding from deeply penetrating, excavated, or fibrotic ulcer with high-risk stigmata; bleeding in which treatment is high risk because thermal therapy will cause perfora- tion or a preexisting perforation could not be ruled out; or bleeding when endoscopic therapy using mechanical approach or radiological approach was unsuccessful. At present, the standard of treatment for HR-AO bleeding is application of contact thermocoagulation or hemostatic clip [1,2]. Unfortunately, about $20 \%$ to $40 \%$ of cases fail or even develop complication from standard therapy $[3,4]$. Hence, there is an urgent need for a safe and more effective endoscopic treatment modality for HR-AO bleeding cases.

The over-the-scope clip (OTSC), as opposed to the standard through-the-scope clip, clamps a large circumferential area of 
tissue securely and deeply [5]. Availability of the OTSC thus provides an opportunity to improve treatment outcome for $\mathrm{HR}-\mathrm{AO}$ bleeding. Indeed, emerging data from Europe on use of OTSC as first-line or second-line treatment in HR-AO bleeding has supported this potential $[6,7]$. However, further data are needed on the efficacy of OTSC in HR-AO bleeding. In addition, bleeding from peptic ulcer disease is still a major issue in Asia, and the role of OTSC is unknown here [8]. In this study, we aimed to determine the safety and efficacy of OTSC to treat HR-AO bleeding in our unit in Singapore.

\section{Patients and methods}

We included all patients treated using OTSC for acute NVUGIB at the Singapore General Hospital from January 2015 to October 2017. We obtained approval from our Institutional Review Board to conduct this study.

We extracted data on demographics, clinical severity, endoscopy findings and OTSC treatment outcome from our endoscopy database for analysis. We used the following definitions [6]:

1. First-line versus second-line treatment:

a) First-line treatment: OTSC used as primary treatment.

b) Second-line treatment: OTSC used after failure of standard endoscopic therapy (clips, thermal coagulation, epinephrine injection, and hemostatic powder)

2. Technical success versus failure

a) Technical success: We successfully deployed an OTSC, and it captured the bleeding vessel. Successful deployment can lead to complete hemostasis or incomplete hemostasis.

b) Technical failure: We deployed an OTSC, and it did not capture the bleeding vessel.

3. Complete hemostasis versus incomplete hemostasis

a) Complete hemostasis: the complete cessation of bleeding after OTSC placement

b) Incomplete hemostasis: continued bleeding after OTSC placement

4. Clinical success versus rebleeding:

a) Clinical success: no rebleeding within 30 days after placement of OTSC.

b) Rebleeding: recurrent bleeding from the treated lesion within 30 days after placement of OTSC.

\section{Learning the OTSC system}

Two endoscopists, Ravishankar Asokkumar, a general gastroenterologist, and Roy Soetikno, a therapeutic endoscopist, performed the procedures. They standardized their technique of deploying the OTSC, with and without using the OTSC anchor, on a thin slab of meat [9]. They started applying OTSC as firstline treatment after experiencing improved outcomes with its use in high-risk NVUGIB lesions $[9,10]$.

\section{Treatment of bleeding}

After resuscitation, we performed the procedures under conscious or deep sedation using midazolam and fentanyl, depending on the clinical situation. We used a therapeutic upper endo- scope (GIF 1TQ160, GIF 1TH190, or GIF 1XTQ160, Olympus Corp, Japan) equipped with water jet function and attached a distal cap (Olympus Corp, Japan) when required. After suctioning blood and identifying the source of bleeding, we equipped the scope with a medium-sized (12-mm) atraumatic OTSC device and clamped the bleeding vessel.

The technique of OTSC application is similar to that of endoscopic variceal ligation. We positioned the OTSC cap such that the bleeding lesion was located inside the perimeter of the cap. Upon achieving a satisfactory position, we applied suction and securely placed the clip deep below the vessel ( $\mathbf{F i g . 1}$ ). In fibrotic ulcers, we used the OTSC anchor to hook the ulcer base and, while applying full suction, pull it inside the cap before clipping.

\section{Control groups}

Rockall Study cohort

We used the study by Rockall and colleagues, who prospectively analyzed the outcomes of 4142 patients with acute upper gastrointestinal bleeding (UGIB) from 74 hospitals in the United Kingdom in 1993 [11]. The patients included in this study were older than 60 years, and most were men (57\%). Their significant comorbidities included ischemic heart disease (16\%), cardiac failure (9\%), liver failure (4\%), disseminated malignancy $(4 \%)$ and renal failure $(3 \%)$. Seven percent $(n=278)$ were receiving anticoagulants at the time of bleeding. The most common diagnosis was peptic ulcer disease $(35 \%, \mathrm{n}=1450)$ and almost half ( $47 \%$ ) of these patients had major stigmata of recent hemorrhage. The endoscopic therapy used in the period of the Rockall study is comparable to the current day standard hemostatic techniques [12].

The researchers developed a complete Rockall risk score (score range, $0-11$ ) based on clinical characteristics and endoscopic findings from 2,956 patients and showed that the mortality rate and the rebleeding rate increased as the risk score increased (see table IVB in Rockall's study, [11]). Since then several studies have validated this risk score and supported its utility in NVUGIB $[13,14]$. For comparison, we classified this cohort into three groups: high-risk ( $R S \geq 8, n=190)$ group, intermediate-risk ( $R S=4$ to $7, n=1,560$ ), and low-risk ( $R S \leq 3, n=$ 1,206).

\section{Our institution's NVUGIB study}

For the second control, we used data from patients with NVU$\mathrm{GIB}$ that were collected prospectively by Ngu and colleagues at the Singapore General Hospital and included data from those with peptic ulcer bleeding $(n=52)$ [15]. The mean age of the patients was 66 years and the majority were men (69\%). Their comorbidities included renal failure $(27 \%)$, ischemic heart disease ( $23 \%)$, malignancy $(4 \%)$, cardiac failure $(2 \%)$, liver failure $(0 \%)$, and other diseases (11\%). At the time of bleeding, about $4 \%$ of patients were treated with anticoagulants, and $33 \%$ were treated with antiplatelet agents. The mean complete Rockall score was 3.9.

Bleeding occurred from a gastric ulcer in 28 patients (54\%) and from a duodenal ulcer in 24 patients $(46 \%)$. These patients 

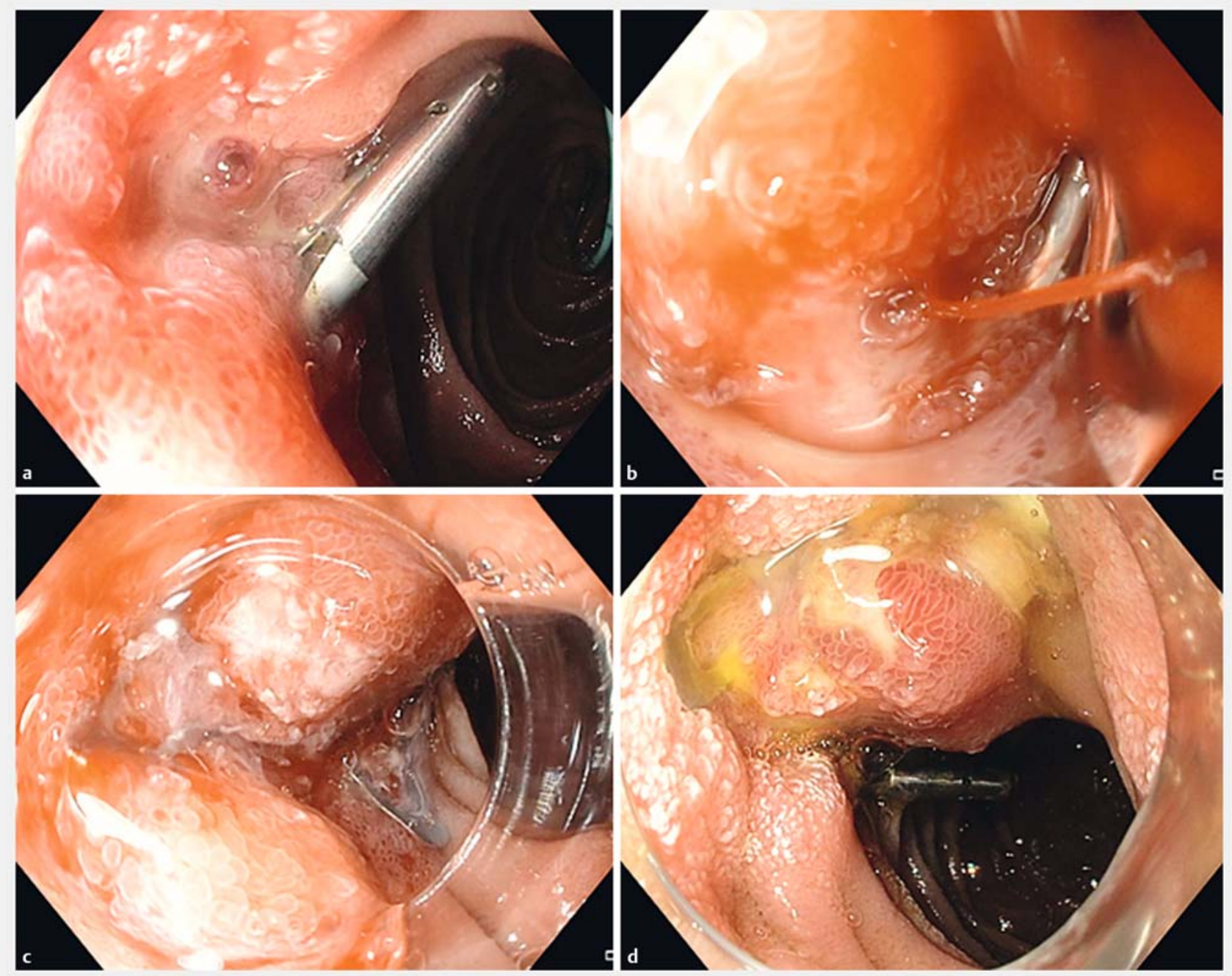

- Fig. 1 Use of OTSC as a second-line treatment modality for a bleeding duodenal ulcer. a A visible artery was seen proximal to a previously deployed clip. b The endoscope was equipped with an OTSC. Active bleeding occurred during suction. $\mathbf{c}$ The bleeding vessel was placed in the center of the OTSC cap and using the suction technique, the OTSC was deployed. The bleeding stopped completely. $\mathbf{d}$ There was no recurrent bleeding. The clip 24 hours later.

were treated using hemoclips or thermal coagulation alone, with or without epinephrine injection. Because of the low number of patients in each Rockall risk category, we classified them into two groups for analysis: intermediate-to-high risk group ( $R S \geq 4, n=29$ ), and low-risk group ( $R S \leq 3, n=23$ ).

\section{Statistical analysis}

The primary outcome measures were rebleeding and mortality rate. We compared our study outcomes with those of the original Rockall cohort and our institution's prospective NVUGIB study.

We performed statistical analysis using IBM SPSS Statistics 23.0 software. We used descriptive statistics to compare continuous variables and Fisher exact test to compare categorical variables.

\section{Results}

\section{Patient characteristics}

Eighteen patients underwent endoscopic hemostasis as inpatients using OTSC on 19 bleeding lesions ( $\triangleright$ Table 1 ). The mean age was $68 \pm 15.9$ years (range, $22-91$ ) and most patients were men $(67 \%)$. More than half of the patients were ASA III (56\%). Ten patients were taking either aspirin $(n=2)$, clopidogrel $(n=4)$, low-molecular-weight heparin $(n=2)$ or a combination of these drugs $(n=2)$ at the time of bleeding. The bleeding occurred from duodenal ulcer (47\%), Dieulafoy's lesion (21\%), gastric ulcer (16\%), and after endoscopic procedures (after gastric biopsy, gastric polypectomy and EUS-FNA of peri-gastric mass) (16\%).

Almost all patients have either intermediate $(63 \%, n=12)$ or high $(32 \%, n=6)$ Rockall risk-score except one with low Rockall risk-score. Ten patients had active bleeding, 5 had a visible ves- 
- Table 1 Clinical characteristics of the patients.

\begin{tabular}{|c|c|}
\hline & Patients $(n=18)$ \\
\hline Age $\pm S D$, years (range) & $68 \pm 15.9(22-91)$ \\
\hline Male, $n$ & $12(67 \%)$ \\
\hline \multicolumn{2}{|l|}{ ASA Class, n (\%) } \\
\hline - Class II & $8(44 \%)$ \\
\hline - Class III & $10(56 \%)$ \\
\hline \multicolumn{2}{|l|}{ Comorbidities } \\
\hline - Ischemic heart disease & $6(33 \%)$ \\
\hline - Chronic kidney disease & $6(33 \%)$ \\
\hline - Atrial fibrillation & $2(11 \%)$ \\
\hline - Diabetes Mellitus & $6(33 \%)$ \\
\hline \multicolumn{2}{|l|}{ Medications, n (\%) } \\
\hline - Aspirin & $2(11 \%)$ \\
\hline - Clopidogrel & $4(22 \%)$ \\
\hline - LMWH & $2(11 \%)$ \\
\hline - Combination treatment & $2(11 \%)$ \\
\hline \multicolumn{2}{|l|}{ Severity score \pm SD, (range) } \\
\hline - Rockall Score & $6.7 \pm 1.3(3-9)$ \\
\hline \multicolumn{2}{|l|}{ Cause of bleeding, $(n=19, \%)$} \\
\hline - Gastric ulcer & $3(16 \%)$ \\
\hline - Duodenal ulcer & $9(47 \%)$ \\
\hline - Dieulafoy’s lesion & $4(21 \%)$ \\
\hline - Recent-GI Intervention & $3(16 \%)$ \\
\hline Hemoglobin \pm SD, g/dL (range) & $8 \pm 2(3-11.7)$ \\
\hline Transfusion \pm SD, units (range) & $1.5 \pm 1.2(0-4)$ \\
\hline
\end{tabular}

sel, and 4 had an adherent clot ( $\triangleright$ Table 2 ). Almost all of them ( $95 \%, n=18$ ) were HR-AO bleeding lesions. Nine (47\%) were chronic and fibrotic ulcers with high-risk stigmata. The remainder either had a large-caliber vessel or were located in the major arterial territory. The mean hemoglobin was $8 \pm 2 \mathrm{~g} / \mathrm{dL}$ (range, $3-11.7$ ). The mean number of packed red cell transfused was $1.5 \pm 1.2$ units (range, $0-4$ ).

\section{Treatment using OTSC}

We used single OTSC to achieve hemostasis in 15 (84\%) patients. In three patients, we had to use two OTSC to secure hemostasis. Ten (53\%) received OTSC as first-line treatment, and in the rest, OTSC was used as a second-line treatment after failed standard therapy. We deployed the OTSC utilizing the suction technique in 17 cases. In two cases, we used the OTSC anchor because the base of the ulcer was excavated and fibrotic ( Table 3).
- Table 2 Characteristics of bleeding lesions.

\begin{tabular}{|c|c|}
\hline & Bleeding lesions $(n=19)$ \\
\hline \multicolumn{2}{|l|}{ Stigmata of bleeding, $n$ (\%) } \\
\hline - Active bleeding & $10(53 \%)$ \\
\hline - Visible vessel & $5(26 \%)$ \\
\hline - Adherent clot & $4(21 \%)$ \\
\hline High risk- adverse outcome bleeding & $18(95 \%)$ \\
\hline $\begin{array}{l}\text { - Large caliber vessel or location in } \\
\text { major arterial territory }\end{array}$ & $15(83 \%)$ \\
\hline - Chronic fibrotic ulcers & $9(50 \%)$ \\
\hline Previous endoscopic treatment & $9(47 \%)$ \\
\hline - Hemoclips & $8(89 \%)$ \\
\hline - Thermal therapy & $2(22 \%)$ \\
\hline - Epinephrine & $7(78 \%)$ \\
\hline - Hemostatic powder & $1(11 \%)$ \\
\hline - Embolization & $1(11 \%)$ \\
\hline
\end{tabular}

Table 3 Outcome of OTSC treatment.

\begin{tabular}{|l|c|}
\hline & Bleeding lesions $(\mathbf{n}=\mathbf{1 9})$ \\
\hline OTSC use, $\mathrm{n}(\%)$ & \\
\hline - First-line treatment & $10(53 \%)$ \\
\hline - Second-line treatment & $9(47 \%)$ \\
\hline OTSC accessories use & 2 \\
\hline Technical success, $\mathrm{n}(\%)$ & $19(100 \%)$ \\
\hline Complete hemostasis, $\mathrm{n}(\%)$ & $19(100 \%)$ \\
\hline Clinical success, $\mathrm{n}(\%)$ & $19(100 \%)$ \\
\hline Rebleeding, $\mathrm{n}$ & 0 \\
\hline Overall mortality, $\mathrm{n}(\%)$ & $3(17 \%)$ \\
\hline Bleeding-related mortality, $\mathrm{n}$ & 0 \\
\hline OTSC, over-the-scope clip & \\
\hline
\end{tabular}

\section{Outcomes}

We achieved technical success in all patients at the end of index endoscopy. We had an initial technical failure in three cases (16\%). Using a second OTSC, we successfully captured the bleeding vessel. We observed incomplete hemostasis after OTSC deployment in 6 patients (33\%), but, in the same settings, we applied additional techniques and attained complete hemostasis in all patients. We achieved clinical success without rebleeding in all patients ( $\downarrow$ Table 3 ). We did not find any difference in the outcome between the first-line and the second-line OTSC treatment $(P=1)$.

Three (17\%) patients died during hospitalization from their comorbidities. The first patient (57 years) had a prolonged in- 
- Table 4 Incomplete hemostasis after OTSC

Bleeding lesions $(n=6)$

Location

\begin{tabular}{|l|c|}
\hline - Stomach & $4(67 \%)$ \\
\hline - Duodenum & $2(33 \%)$ \\
\hline HR-AO bleeding & $5(83 \%)$ \\
\hline Cause & \\
\hline - OTSC-related & $2(33 \%)$ \\
\hline - Bleeding stigmata-related & $3(50 \%)$ \\
\hline - Both & $1(17 \%)$ \\
\hline Additional combination therapy & $4(67 \%)$ \\
\hline - Hemoclips & $3(75 \%)$ \\
\hline - Thermal therapy & $2(50 \%)$ \\
\hline - Epinephrine & $3(75 \%)$ \\
\hline - N-butyl cyanoacrylate glue & $1(25 \%)$ \\
\hline - Prophylactic embolization & $1(25 \%)$ \\
\hline Complete hemostasis, n (\%) & $6(100 \%)$ \\
\hline Rebleeding, n & 0 \\
\hline
\end{tabular}

patient stay ( 3 months) and was treated for infected foot gangrene, fluid overload secondary to cardiac and end-stage renal failure, and recurrent hospital-acquired pneumonia before the episode of NVUGIB. He died 10 days after placement of OTSC while being treated for fluid overload and sepsis. The second patient (84 years) had advanced liver cirrhosis with multifocal hepatocellular carcinoma. The third patient was an elderly female (91 years) undergoing treatment for a hip fracture. She died suddenly 3 days after treatment of bleeding using OTSC. None of these patients had clinical signs of rebleeding or a significant drop in hemoglobin $(>2 \mathrm{~g} / \mathrm{dL})$ at the time of demise.

\section{Incomplete hemostasis after OTSC placement}

Six patients had continued bleeding despite OTSC placement: 2 (33\%) duodenal ulcer, $1(17 \%)$ gastric ulcer, $1(17 \%)$ gastric Dieulafoy's lesion and 2 (33\%) after gastric biopsy and EUSFNA of peri-gastric mass ( $\downarrow$ Table 4 ). Five of the 6 patients were actively bleeding during endoscopy. Their mean Rockall score was $7 \pm 1.2$. We used OTSC as first-line treatment in four patients and as second-line treatment in the rest.

Of the six patients with continued bleeding, two were OTSCrelated, three were bleeding stigmata-related, and one had a combination of both factors. In the two OTSC-related patients, the OTSC was misplaced in a chronic fibrotic ulcer, causing bleeding. We stopped the bleeding using a second successful OTSC ( $\triangleright$ Fig. 2). In the three bleeding stigmata-related patients, we observed one had a large-caliber vessel, one had a chronic fibrotic ulcer, and the other was taking clopidogrel. Although the OTSC was well placed in all three, the bleeding con- tinued, albeit at a slower rate. We applied additional therapy (clips, epinephrine, thermal therapy) and stopped the bleeding completely. In the last patient, we released the OTSC prematurely, resulting in superficial placement. We removed it and placed a second OTSC successfully. However, the bleeding continued from the large-caliber vessel. We had to inject epinephrine, use clips and glue to stop the bleeding completely.

In one case (Dieulafoy's lesion), despite complete hemostasis, we performed additional prophylactic embolization because of the large caliber of the vessel.

\section{Comparison to the Rockall cohort}

Using the complete Rockall-risk score, we stratified our cohort into high-risk $(n=6)$, intermediate-risk $(n=12)$, and low-risk ( $n$ $=1$ ) groups and compared our outcomes with the original Rockall study $(n=2,956)$.

In the high-risk group, we found the rebleeding rate was significantly lower with OTSC as compared to similar patients receiving standard treatment in the Rockall study (0\% vs. $53 \%, P$ $<0.01$ ) ( $\vee$ Fig. 3). In the intermediate-risk group, we observed OTSC reduced the rebleeding rate compared to standard treatment in the Rockall study ( $0 \%$ vs. $24 \%, P<0.08$ ). In the low-risk group, there was no difference in rebleeding rates between OTSC and the standard treatment used in the Rockall study ( $0 \%$ vs. $7 \%, P=1)$. We did not observe lower mortality rates in any of the three groups as compared to those reported by the Rockall study ( $\triangleright$ Table 5 ).

\section{Comparison to our Institution NVUGIB study}

We categorized our cohort into two groups, intermediate-tohigh risk $(n=18)$ and low-risk $(n=1)$, based on the complete Rockall-risk score, and compared our outcomes with our institution's NVUGIB study.

We found OTSC decreased the rebleeding rate in the intermediate-to-high risk group compared to similar patients receiving standard treatment in our institution's NVUGIB study ( $0 \%$ vs. $21 \%, P=0.06$ ). In the low-risk group, OTSC did not change the rebleeding rate as compared to the standard treatment in our institutional study ( $0 \%$ vs. $9 \%, P=1)$. There was no difference in the mortality rate between the two cohorts irrespective of their risk scores ( $\triangleright$ Table 5 ).

\section{Complications}

There were no complications associated with OTSC placement.

\section{Discussion}

We report the outcome of OTSC in bleeding lesions with HR-AO features. OTSC was successful in achieving complete hemostasis without rebleeding in all patients either as the primary or secondary treatment. In addition, we described the causes and solutions to incomplete hemostasis after deployment of OTSC. Despite successful hemostasis, we observed a $17 \%$ mortality rate, which was due to patients' comorbid illnesses.

We lend strong support to the recently published studies showing superior efficacy of OTSC as compared to standard therapy in the treatment of HR-AO bleeding. A randomized 

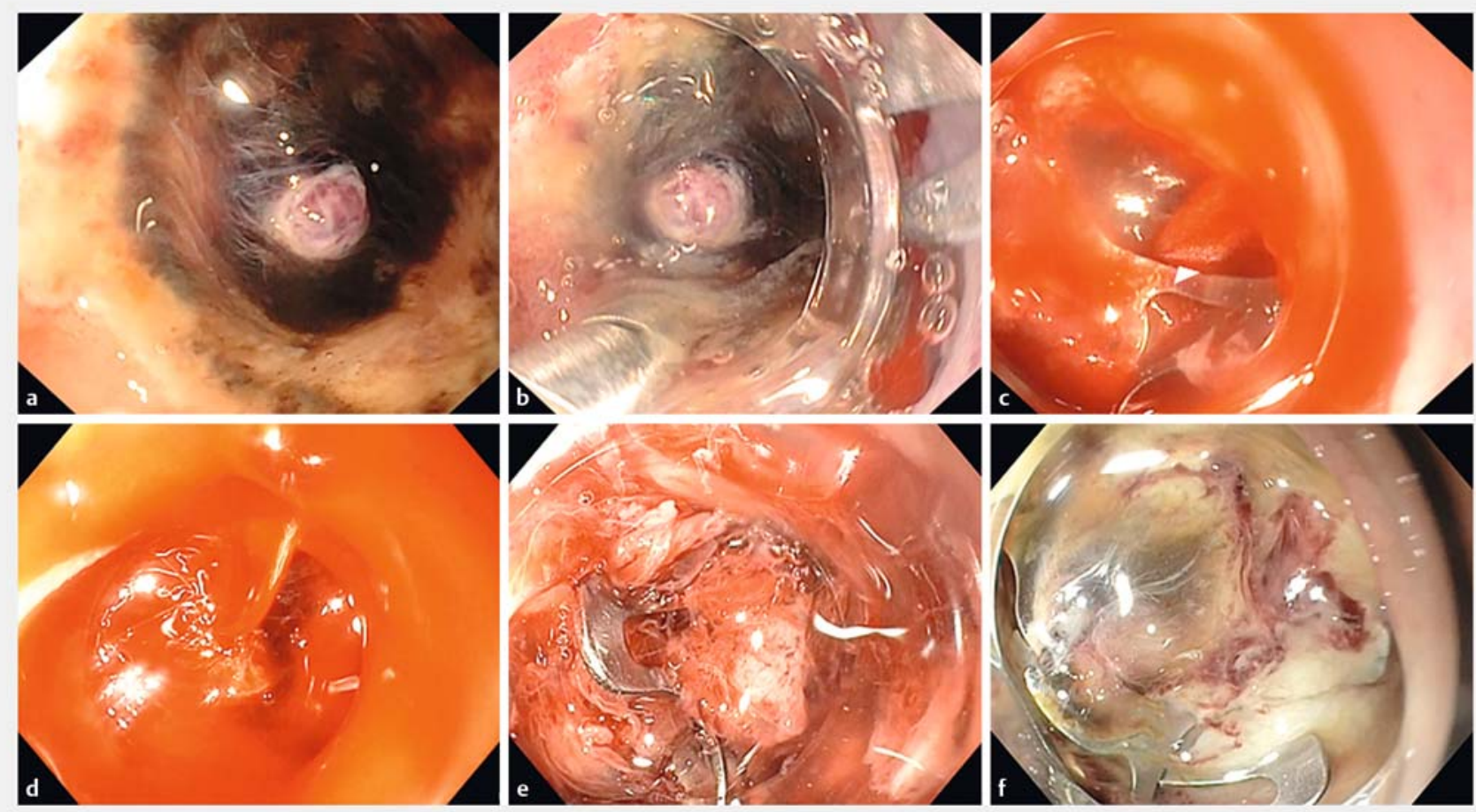

- Fig. 2 Cause and solution of incomplete hemostasis after OTSC placement. a nonsteroidal anti-inflammatory drug-related duodenal ulcer with a large visible vessel was seen in the distal duodenal bulb of an elderly patient with metastatic lung cancer. The ulcer was located within a narrowed lumen. $\mathbf{b}$ A therapeutic endoscope with $6-\mathrm{mm}$ channel that was equipped with a large OTSC was used (OD of OTSC $=21 \mathrm{~mm})$. The tip of the OTSC anchor was placed next to the visible vessel. c After opening the anchor, the ulcer was pulled and simultaneously suctioned into the OTSC, and the clip was released. $\mathbf{d}$ A large stream of blood was seen flowing from the artery. The clip was misplaced. The endoscope was left to suction the blood to prevent formation of large clots and to maintain visualization, while another therapeutic endoscope was being equipped with a medium-size OTSC. e The bleeding vessel was suctioned into the OTSC. The clip was placed ideally. Bleeding ceased instantly. f There was no further bleeding. The clip at 24 hours.

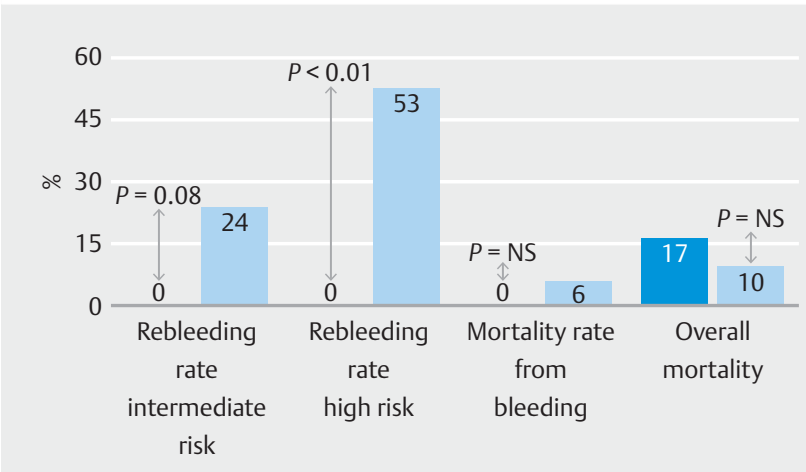

- Fig. 3 Results of our study as compared to original Rockall study. Use of OTSC significantly reduced risk of rebleeding in the highrisk Rockall score patients. A trend toward decreased rebleeding was seen in the intermediate-risk Rockall score patients. The mortality rate from bleeding or from all causes was similar to the original Rockall study.

study, which has been published only in abstract form, showed OTSC $(84.4 \%)$ was superior to standard therapy $(40.6 \%)$ in achieving hemostasis $(P<0.001)$ [16]. A multicenter retrospective study showed that first-line use of OTSC, alone or in combi- nation with additional hemostatic techniques, was significantly superior in preventing rebleeding and rebleeding-related mortality in the intermediate and high-risk cases as compared to the standard therapy in original Rockall data [17].

We observed incomplete hemostasis after placement of OTSC in some cases. A recent retrospective study assessing utility of OTSC in HR-AO lesions showed recurrent bleeding (19\%) from the OTSC site within 5 days [18]. A similar finding was also observed in other studies [16,19-21]. The causes for these findings were not described, but we suspect that incomplete hemostasis after OTSC may have resulted in recurrent bleeding. We have identified three common patterns that resulted in OTSC failure: 1) delayed closure of OTSC occurring in lesions with large caliber artery and those with deep fibrotic base; 2) shallow placement of OTSC resulting from inadequate suction or premature clip deployment; and 3) misplacement of OTSC because of poor visualization, difficult anatomy, and unstable endoscope position. We were able to address these issues and observed no recurrent bleeding ( $\triangleright$ Fig.4). Understanding the causes of incomplete hemostasis and recurrent bleeding may further improve efficacy of OTSC use in treatment of HR-AO bleeding.

Our study is limited by its retrospective design and small sample size. In addition, the procedures were performed by 
- Table 5 Comparison of OTSC outcome among different groups.

\begin{tabular}{|c|c|c|c|c|c|c|}
\hline \multirow[b]{2}{*}{ Rockall Risk Score } & \multicolumn{3}{|l|}{ Overall mortality } & \multicolumn{3}{|l|}{ Overall rebleeding } \\
\hline & $\begin{array}{l}\text { Rockall Cohort } \\
(n=2956)\end{array}$ & $\begin{array}{l}\text { Study Cohort } \\
(n=18)\end{array}$ & $P$ value & $\begin{array}{l}\text { Rockall Cohort } \\
(n=2956)\end{array}$ & $\begin{array}{l}\text { Study Cohort } \\
(n=18)\end{array}$ & $P$ value \\
\hline Low risk (RS $\leq 3$ ) & $9(1 \%)$ & $0(0 \%)$ & ns & $84(7 \%)$ & $0(0 \%)$ & ns \\
\hline Intermediate risk $(\mathrm{RS}=4-7)$ & $203(13 \%)$ & $1(8 \%)$ & ns & $374(24 \%)$ & $0(0 \%)$ & 0.08 \\
\hline \multirow[t]{2}{*}{ High risk (RS $\geq 8$ ) } & $78(41 \%)$ & $2(33 \%)$ & ns & $101(53 \%)$ & $0(0 \%)$ & 0.01 \\
\hline & $\begin{array}{l}\text { Institution cohort } \\
(n=52)\end{array}$ & $\begin{array}{l}\text { Study cohort } \\
(n=18)\end{array}$ & & $\begin{array}{l}\text { Institution cohort } \\
(n=52)\end{array}$ & $\begin{array}{l}\text { Study cohort } \\
(n=18)\end{array}$ & \\
\hline Low risk (RS $\leq 3$ ) & $0(0 \%)$ & $0(0 \%)$ & ns & $2(9 \%)$ & $0(0 \%)$ & ns \\
\hline Intermediate-to-high risk (RS $\geq 4$ ) & $2(7 \%)$ & $3(17 \%)$ & ns & $6(21 \%)$ & $0(0 \%)$ & 0.06 \\
\hline
\end{tabular}

ns, not significant; RS, Rockall score.
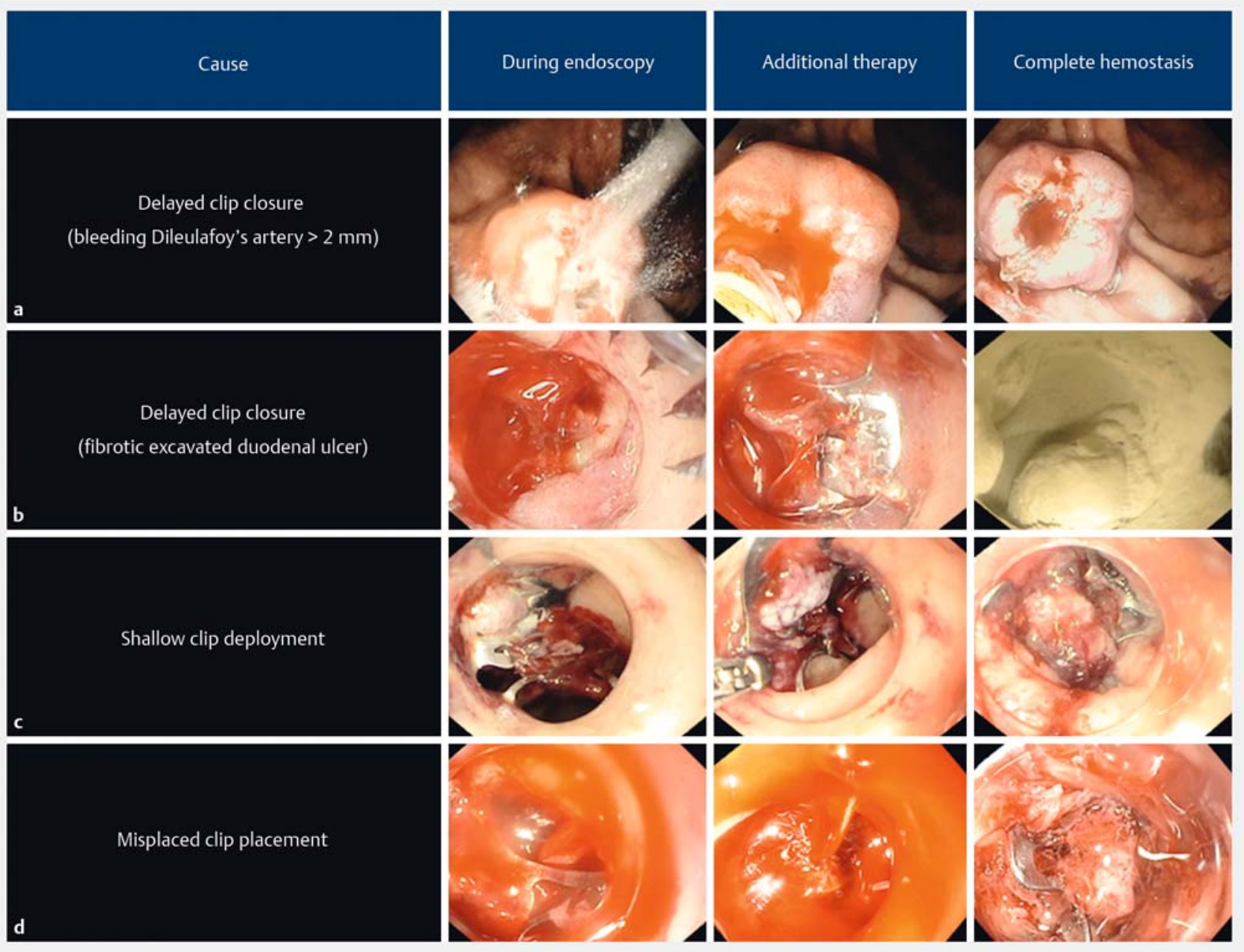

- Fig. 4 Classification of causes for incomplete hemostasis after OTSC. a Delayed compression of a large-caliber Dieulafoy's vessel resulting in continued bleeding. Using additional thermal therapy, the bleeding vessel was coagulated and complete hemostasis was achieved. b Delayed compression of high-risk stigmata within a fibrotic duodenal ulcer. Using additional application of hemostatic powder, we achieved complete hemostasis. c Shallow placement of OTSC in duodenal ulcer resulting in continued bleeding. The clip was pulled and removed using forceps, and with a second OTSC, the bleeding was stopped. $\mathbf{d}$ Misplacement of OTSC in the narrow lumen of the duodenum, causing massive bleeding. Using a second OTSC, we crossed the first clip and clamped the bleeding vessel achieving permanent hemostasis. 
only two endoscopists. However, patients with HR-AO bleeding were not common. To validate our study findings further, we compared our outcomes with the original Rockall study and a historical control group. Prior to embarking on conducting a randomized study, we needed to understand the causes and develop solutions for incomplete hemostasis after placement of OTSC

Our data add further evidence about the superior efficacy and safety of OTSC in the treatment of HR-AO NVUGIB. We suggest using OTSC as the first-line treatment in high-risk Rockall score patients with HR-AO lesions. We, like others, encountered occasional incomplete hemostasis after OTSC placement. However, in our study, albeit small, we showed that further endoscopic therapy could achieve complete hemostasis. A formal cost-effectiveness analysis is needed to determine the impact of first-line use of OTSC in treatment of HR-AO bleeding. In addition, studies to document that OTSC can lead to similarly high efficacy in the community setting are also desirable.

Competing interests

None

References

[1] Barkun AN, Bardou M, Kuipers E] et al. International consensus recommendations on the management of patients with nonvariceal upper gastrointestinal bleeding. Ann Intern Med 2010; 152: 101 - 113

[2] Gralnek IM, Dumonceau JM, Kuipers E] et al. Diagnosis and management of nonvariceal upper gastrointestinal hemorrhage: European Society of Gastrointestinal Endoscopy (ESGE) Guideline. Endoscopy 2015; 47: a1 - 46

[3] Laine L, Jensen DM. Management of patients with ulcer bleeding. Am J Gastroenterol 2012; 107: 345-360

[4] Barkun AN, Martel M, Toubouti Y et al. Endoscopic hemostasis in peptic ulcer bleeding for patients with high-risk lesions: a series of meta-analyses. Gastrointest Endosc 2009; 69: 786 - 799

[5] Kato M, Jung Y, Gromski MA et al. Prospective, randomized comparison of 3 different hemoclips for the treatment of acute upper GI hemorrhage in an established experimental setting. Gastrointest Endosc 2012; 75: 3-10

[6] Richter-Schrag H], Glatz T, Walker C et al. First-line endoscopic treatment with over-the-scope clips significantly improves the primary failure and rebleeding rates in high-risk gastrointestinal bleeding:
A single-center experience with 100 cases. World J Gastroenterol 2016; 22: 9162 - 9171

[7] Wedi E, Gonzalez S, Menke D et al. One hundred and one over-thescope-clip applications for severe gastrointestinal bleeding, leaks, and fistulas. World J Gastroenterol 2016; 22: 1844 - 1853

[8] Lau JY, Sung J, Hill C et al. Systematic review of the epidemiology of complicated peptic ulcer disease: incidence, recurrence, risk factors and mortality. Digestion 2011; 84: $102-113$

[9] Asokkumar R, Kaltenbach T, Soetikno R et al. Use of over-the-scope clip to treat bleeding duodenal ulcers. Gastrointest Endosc 2016; 83: 459-460

[10] Asokkumar R, Soetikno R. Misplaced "bear claw" in a bleeding gastric ulcer: What next? Gastrointest Endosc 2016; 84: 366 - 367

[11] Rockall TA, Logan RF, Devlin HB et al. Risk assessment after acute upper gastrointestinal hemorrhage. Gut 1996; 38: 316-321

[12] Rauws EA, Kool G, Bolwerk C. New approaches to endoscopic therapy for a haemostasis upper GI bleed. Scand J Gastroenterol Suppl 1996; 218: $116-123$

[13] Wang CY, Qin J, Wang J et al. Rockall score in predicting outcomes of elderly patients with acute upper gastrointestinal bleeding. World J Gastroenterol 2013; 19: 3466-3472

[14] Kim BJ, Park MK, Kim S] et al. Comparison of scoring systems for the prediction of outcomes in patients with nonvariceal upper gastrointestinal bleeding: a prospective study. Dig Dis Sci 2009; 54: 2523 2529

[15] Stanley AJ, Laine L, Dalton HR et al. Comparison of risk scoring systems for patients presenting with upper gastrointestinal bleeding: international multicentre prospective study. BMJ 2017; 356: i6432

[16] Schmidt A, Goelder S, Messmann $\mathrm{H}$ et al. Over-the-scope-clips versus standard endoscopic therapy in patients with recurrent peptic ulcer bleeding and a prospective randomized, multicentre trial (Sting). Gastrointest Endosc 2017; 85: AB50

[17] Wedi E, Fischer A, Hochberger J et al. Multicenter evaluation of firstline endoscopic treatment with the OTSC in acute non-variceal upper gastrointestinal bleeding and comparison with the Rockall cohort: the FLETRock study. Surg Endosc 2018; 32: 307 - 314

[18] Brandler J, Baruah A, Zeb M et al. Efficacy of over-the-scope clips in management of high-risk gastrointestinal bleeding. Clin Gastroenterol Hepatol 2018; 16: 690-696.e1

[19] Chan SM, Chiu PW, Teoh AY et al. Use of the Over-the-scope clip for treatment of refractory upper gastrointestinal bleeding: a case series. Endoscopy 2014; 46: 428-431

[20] Lamberts R, Koch A, Binner C et al. Use of over-the-scope clips (OTSC) for hemostasis in gastrointestinal bleeding in patients under antithrombotic therapy. Endosc Int Open 2017; 5: E324-E330

[21] Wedi E, von Renteln D, Gonzalez S et al. Use of the over-the-scopeclip (OTSC) in non-variceal upper gastrointestinal bleeding in patients with severe cardiovascular comorbidities: a retrospective study. Endosc Int Open 2017; 5: E875-E882 